\title{
A Geomulticast Architecture and Analysis Model for Ad-Hoc Networks
}

\author{
Beongku $\mathrm{An}^{1}$ and Dohyeon $\mathrm{Kim}^{2}$ \\ ${ }^{1}$ School of Electronic, Electrical and Computer Engineering, Hongik University \\ Jochiwon, Chungnam, Korea, 339-701, Tel.:041-860-2243 \\ beongkulwow. hongik.ac.kr \\ ${ }^{2}$ School of Information and Communications, Cheonan University \\ Cheonan-City, Chungnam, Korea, 330-180, Tel.:041-620-9418 \\ dhkim@cheonan.ac. kr
}

\begin{abstract}
In this paper, we propose a geomulticast architecture and analysis model for ad-hoc networks. We define and formulate the probability of geomulticast error and geomulticast error cost. Based on the geomulticast architecture and analysis model, we present how to evaluate the performance of the geomulticast architectures and protocols, and we obtain some insight about the impact of the design and operational parameters on the system performance.
\end{abstract}

\section{Introduction}

Geomulticast is a specialized location-dependent multicasting technique, where messages are multicast to some specific user groups within a specific geographical zone. While conventional multicast protocols define a multicast group as a set of nodes with a multicast address and geocast[1,2] defines a geocast group as all the nodes within a specified zone at a given time, a geomulticast group is defined as a set of nodes of some specific groups within a specified zone. In general, geomulticast combines geocast and multicast for dynamic message delivery to a specific group within a geomulticast zone. If a specific geographic zone is very large and there are many different kind of groups within this zone, we do not need to broadcast to all nodes within the specific zone. In this paper, we propose a geomulticast architecture and analysis model to support geomulticast services for performance analysis and evaluation in the challenging environment of mobile ad-hoc wireless networks. We define and formulate the probability of geomulticast error and geomulticast error cost in order to evaluate the performance of the design alternatives and protocols used for the support of geomulticast services in mobile ad-hoc wireless networks.

\section{Geomulticast Architecture and Analysis Model}

To support geomulticast, it is first needed to define the geomulticast zone formulation method and geomulticast zone representation method. We use several zone formulation methods that range from simple specific zone formulation techniques (i.e., circle 
with given radius) to the most general cases, while we use several approximation methods for geomulticast zone representation to reduce routing overhead as follows: circular representation, elliptical representation, and rectangular representation. Fig. 1 presents a high level description of the proposed network architecture for supporting geomulticast using clustering[3] as underlying structure in mobile ad-hoc wireless networks. The key idea of the proposed network structure is the extension of cellular network concepts into mobile ad-hoc wireless networks to support geomulticast services in a stable and cost-effective way. Our network structure for supporting geomulticast services consists of the following elements and components: Geomulticast Control Office (GeoCO), clusterheads ( $\mathrm{CHs}$ ), mobile nodes (MNs). The functions performed by $\mathrm{CH}$ and $\mathrm{GeoCO}$ are similar to those performed by BS (base station) and MSC (mobile switching center) respectively in cellular networks. In our structure, the source clusterhead $(\mathrm{CH})$ plays the role of GeoCO. The main function of $\mathrm{GeoCO}$ is the membership management (i.e., geomulticast group construction and maintenance).

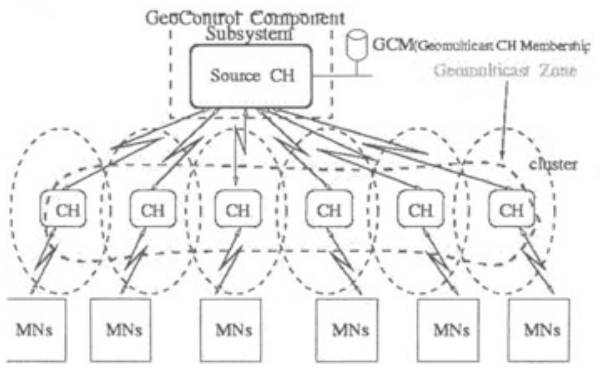

Fig. 1. Network architecture

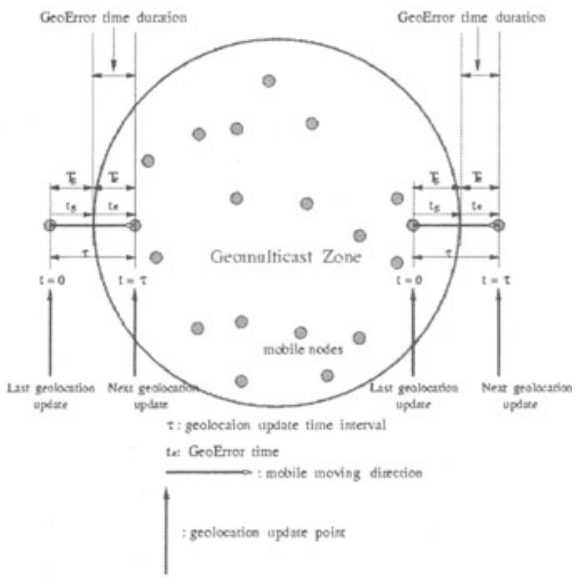

Fig. 2. The model

Next, we define and formulate a framework model in order to evaluate the performance of the design alternatives, algorithms and implementations used for geomulticast services in mobile ad-hoc wireless networks. Since the main objective of our efforts is to design a modeling framework for performance analysis and evaluation, we introduce and define the probability of geomulticasting error (GeoError), Pge, and the Geomulticast Packet Delivery Ratio(GPDR) as performance measures. The probability of GeoError (Fig. 2), Pge, refers to the probability of occurrence of either of the following events: a) a mobile that is inside the geomulticast zone does not receive the geomulticast message for some time, and b)a mobile that is not in the geomulticast zone does receive the geomulticast message for some time. Let us denote by $\tau$ the geolocation update time interval and by $\mathrm{T}_{e}$ the GeoError time interval for a specific mobile due to either one of the two events described above (see fig. 2).

From the above discussion, it is obvious that the rate of geomulticast zone boundarycrossing, $\lambda_{c}$, before the geomulticast membership list is updated is one of the most significant contributors to the total probability of GeoError, especially when 
the geomulticast membership decision is made at the network level. Here, we develop a framework in order to evaluate the effects and associated trade offs, and we gain some insight about the optimum location update interval through a numerical study. Specifically, we study the effect of the mobile node geolocation periodic update interval on the packet delivery accuracy and the associated overhead (signaling load). This model assumes that the mobile nodes move with constant velocity during time interval $\tau$ in a random direction $\theta$ that is uniformly distributed in $[0,2 \pi]$. Considering a geomulticast area with perimeter $S$ where $\rho$ mobile terminals per unit area are located, the rate of geomulticast zone boundary crossing per unit time (average number of mobile nodes crossing the area border per unit of time), denoted by $\lambda_{c}$ is where $\beta$ denotes the activity ratio of mobiles (in the following without loss of generality, we may assume $\beta=1$ ), and $V$ is the average mobile node velocity. For example, if the geomulticast zone is represented by a circle with radius $R$ then $\lambda_{c}=2 \rho V R$. In general, $\lambda_{c}$ depends on the actual size of the geomulticast zone, the network density, and the mobility pattern of the mobile nodes (e.g. velocity). We assume that the number of messages that arrive at a mobile node during a time $t$ is a Poisson random variable with $\lambda_{a}$. Moreover, let us denote by te the random variable that represents the GeoError time interval (i.e. the time between the point that a mobile is erroneously considered (or not considered) member of the geomulticast session due to the two events described before, and the point until the next position update). Assuming that the interval between two boundaries crossings of a mobile are much longer than $\tau$, which should be case in any practical system, then te has a uniform distribution in interval $[0, \tau]$. Thus, the probability of $k$ message arrival during time $T e$ is

$$
P_{e}[n=k]=\int_{0}^{\tau} P\left[n=k \mid T_{e}=t\right] f_{e}(t) d t=\int_{0}^{\tau} \frac{e^{-\lambda_{\alpha} t}\left(\lambda_{\alpha} t\right)^{k}}{k !} \frac{1}{\tau} d t
$$

Since as mentioned before te follows a uniform distribution between $[0, \tau]$, we can easily show based on the properties of conditional expectation the average rate of error (E[error packet rate]) message arrival. Then, we can calculate the probability of $\operatorname{GeoError}\left(P_{\mathrm{ge}}\right)$ as follows:

$$
P_{g e}=\frac{E[\text { error packet rate] }}{E[\text { desired packet rate] }}=\frac{2 * \lambda_{a} * \lambda_{c} * \frac{\tau}{2} * h}{\lambda_{a} * B}=\frac{\lambda_{c} * \tau * h}{B}
$$

where $\mathrm{B}$ denotes the average number of nodes within the geomulticast zone that are geomulticast members. Based on the model under consideration, the total number of nodes within a zone of area $\mathrm{F}$ is given by $\rho * \mathrm{~F}$, and therefore ${ }^{\mathrm{h}=\frac{\mathrm{B}}{\rho * \mathrm{~F}}}$. Substituting this in the previous relation, we obtain: $P_{g e}=\frac{\lambda_{c} * \tau}{\rho * F}$. The Geomulticast Packet Delivery Ratio(GPDR) can be defined as: $G P D R=1-P_{g e}$.

In the following, we develop a cost function to quantify the trade off between the penalty associated with the occurrence of errors in packet delivery and the associated cost for supporting certain degree of accuracy in a geomulticast architecture[6]. We 
consider only the cost directly related to the geomulticast session setup, the update of the geolocation and the loss of packets due to the inaccuracy of position information as a result of the node mobility and the gelocation update time interval. Let us denote by Csetup the cost for geomulticast session setup, by Cgeo-update the cost of geolocation update, and by Cerror the GeoError cost per time unit. Then, the corresponding average cost per time unit, Ctotal, is: Ctotal $=$ Csetup + Cgeo-update + Cerror.. . . We denote by a the rate at which geolocation updates are generated by a mobile node(e.g. updates per time unit). Let us denote by $c_{n}, \mathrm{n}=1,2, \ldots$, the cost of loosing $\mathrm{n}$ calls. Then, the corresponding average GeoError cost per time unit, Cerror is:

$$
C_{\text {error }}=2 * \lambda_{c} * h * \sum_{n=1}^{\infty} c_{n} P_{e}[n]
$$

For the sake of simplicity in the following, we can reasonably assume that the cost of loosing the $n_{\text {th }}$ message arrival after geomulticast zone boundary crossing by a mobile node is independent of $\mathrm{n}$. Thus, $\forall n, c_{n}=n c_{1}$. Consequently:

$$
\mathrm{C}_{\text {error }}=2 * \lambda_{c} * h * c_{1} \sum_{n=1}^{\infty} n P_{e}[n]=c_{1} * 2 * \lambda_{c} * h * \lambda_{a} * \frac{\tau}{2}
$$

Finally, we get:

$$
C_{\text {total }}=C_{\text {setup }}+C_{\text {geo-update }}+c_{1} * 2 * \lambda_{c} * h * \lambda_{a} * \frac{\tau}{2}
$$

\section{Performance Evaluation and Discussion}

In this section, we present some numerical results that we obtained based on the analytical framework as well as via modeling and simulation using the Optimized Network Engineering Tool (OPNET).

A mobile ad-hoc network consisting of 50 mobile nodes that are placed randomly within a rectangular region of $1000 \mathrm{~m} \times 1000 \mathrm{~m}$ is modeled for the purposes of this study. The results presented in the following section correspond to the use of circular geomulticast zone with radius $\mathrm{R}(150 \mathrm{~m}-300 \mathrm{~m})$. The mobile nodes are assumed to have constant radio range of $\mathrm{Z}=250 \mathrm{~m}$. Throughout our study, we assume that a link fails, or reappears, as a node goes out or in transmission range of another node, due to the mobility of the nodes. Mobile nodes are assumed to be moving around throughout the network. The speed and the direction of each move are uniformly distributed, with speed range $[0,80 \mathrm{~km} / \mathrm{h}]$ and direction range $[0,2 \pi]$, respectively. We use one geomulticast source node (sender) while the gomulticast members are selected randomly within the geomulticast zone.

One of the main objectives of this numerical evaluation is to compare the geomulticast packet delivery ratio between simulation results and results of analysis using the proposed model. We implement a geomulticast routing protocol[6], namely DGR 


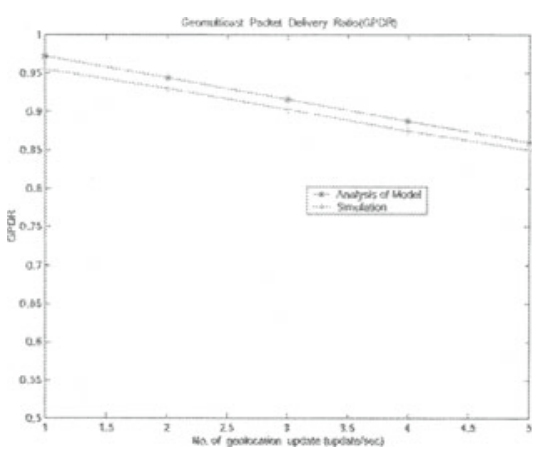

Fig. 3. GPDR as a function of update interval

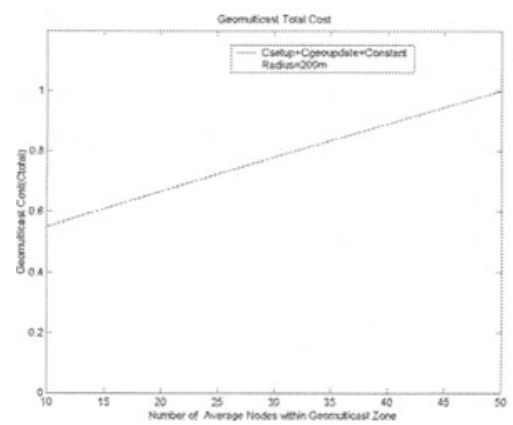

Fig. 5. Ctotal as a function of average number of nodes within geomulticast zone

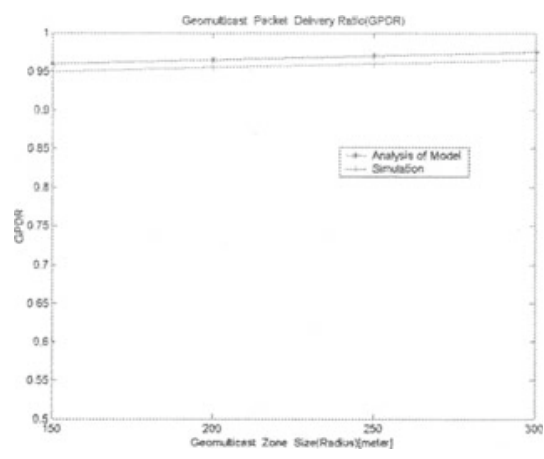

Fig. 4. GPDR as function of geomulticast zone size

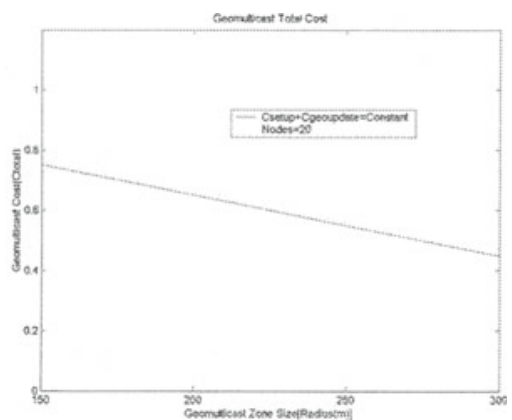

Fig. 6. Ctotal as a function geomultict zone size(Radius)

(direction-guided routing). Fig. 5 presents the geomulticast packet delivery ratio as a function of geolocation update time interval (i.e., $\tau$ ) for mobility $40 \mathrm{~km} / \mathrm{hr}$, packet arrival rate(PAR) 5 packets/sec and geomulticast zone with Radius $250 \mathrm{~m}$. From this figure we can see that geomulticast packet delivery ratio(GPDR) is decreasing with the increase of $\tau$. However, as we discussed before, this is accompanied with a decrease in signaling load due to the position update generation process. Specifically, fig. 4 presents the GPDR as a function of the geomulticast zone size (i.e., radius $R$ ) for mobility $40 \mathrm{~km} / \mathrm{hr}$, PAR 5 packets $/ \mathrm{sec}$ and geolocation update time interval $\tau=1$ sec. As we can see from this figure, the geomulticast packet delivery ratio remains relatively constant with the change of the geomulticast zone size. The main goal of the fig. 5 and figure 6 is the evaluation of the error cost (Cerror) for supporting geomulticast services. Therefore, we assume $C$ setup and $C$ geo-update are constant while $C$ total is normalized as $C$ total $=1$ when Radius is $200 \mathrm{~m}$ and nodes within geomulticast zone is 50 . Fig. 5 presents the total cost as a function of the average number of nodes within the geomulticast zone while fig. 6 presents the total cost as a function of geomulticast zone size. As can be see from these figures(fig. 5 and fig. 6), the total cost 
for geomulticast services is linearly decreased as the geomulticast zone size is increased while the total cost is linearly decreased as the average number of nodes within the geomulticast zone is increased.

The results in figure3-figure6 show the proposed model can be efficiently used as measures of performance analysis and evaluation for supporting geomulticast services in mobile ad-hoc wireless networks.

\section{Conclusion}

In this paper, we have presented a geomulticast architecture and analysis model in order to analyze and evaluate the performance of the design alternatives for algorithms and implementations used for geomulticast services. The probability of GeoError, the geomulticast packet delivery ratio, and GeoError cost are derived and the tradeoff between the geolocation update interval is discussed. The performance evaluation of the modeling framework has demonstrated their efficiency in terms of geomulticast packet delivery ratio(GPDR) as a function of geomulticast zone size, geolocation update time interval. The results in figure3-figure6 show the proposed model can be efficiently used as measures of performance analysis and evaluation for supporting geomulticast services in mobile ad-hoc wireless networks.

\section{References}

1. J.C. Navas and T. Imielinski, "GeoCast - Geographic Addressing and Routing", Proc. of MOBICOM'97, pp.151-160, Budapest, Hungary, September 26-30, 1997.

2. Y.-B. Ko, N.H. Vaidya, "Geocasting in mobile ad-hoc networks: location-based multicast algorithms", Proc. of IWMC-Sa'99, New Orleans, USA, 1999.

3. Beongku An, Symeon Papavassiliou, "A Mobility-Based Approach to Support Mobility Management and Multicast Routing in Mobile Ad-hoc Wireless Networks", The International Journal of Network Management(JNM), vol.11, no.6, pp.387-395, December 2001.

4. I.F. Akyildiz and J.S.M. Ho, "Dynamic mobile user location update for wireless PCS networks", Wireless Networks 1 (1995) 187-196.

5. A. Bar-Noy, I. Kessler and M. Sidi, "Mobile users: To update or not to update ?", Wireless Networks 1 (1995) 175-186.

6. Beongku An, Symeon Papavassiliou, "An architecture for supporting geomulticast services in mobile ad-hoc wireless networks”, Proc. of MILCOM2001, USA, October 2001. 